Gold Configuration File

\title{
POPULATION
}

popsiz $=100$

select_pressure $=1.100000$

$\mathrm{n} \_$islands $=3$

maxops $=30000$

niche_siz $=2$

\section{GENETIC OPERATORS}

pt_crosswt $=95$

allele_mutatewt $=95$

migratewt $=10$

\section{FLOOD FILL}

radius $=10$

origin $=29.359 \quad 4.40462 .343$

do_cavity $=1$

floodfill_atom_no $=0$

cavity_file = cavity.atoms

floodfill_center = point

\section{DATA FILES}

protein_datafile $=$ clipped-site.pdb

ligand_data_file ligand.mol2 1

param_file $=$ DEFAULT

set_ligand_atom_types $=1$

set_protein_atom_types $=0$ 
directory $=$.

tordist_file $=$ DEFAULT

make_subdirs $=0$

save_lone_pairs $=1$

\#fit_points_file = fit_pts.mol2

\#read_fitpts $=0$

\section{FLAGS}

display $=0$

internal_ligand_h_bonds $=0$

n_ligand_bumps $=0$

flip_free_corners $=0$

flip_amide_bonds $=0$

flip_planar_n $=1$

use_tordist $=1$

start_vdw_linear_cutoff $=3$

initial_virtual_pt_match_max $=6$

\section{TERMINATION}

early_termination $=0$

n_top_solutions $=3$

rms_tolerance $=1.5$

\section{CONSTRAINTS}

\section{COVALENT BONDING}

covalent $=0$

\section{MOPAC Parameters}

The command line for the MOPAC program is as follows: 
Where ' $\mathrm{x}$ ' is the charge on the molecule in question. These parameters have the following meanings:

Table 3. Explanation of MOPAC parameters used in QM/MD algorithm

\begin{tabular}{|c|c|}
\hline XYZ & Interpret input file as cartesian coordinates instead of as a Z-matrix \\
\hline $\mathrm{EF}$ & Use eigenvector following energy minimisation method. \\
\hline GEO-OK & $\begin{array}{l}\text { Switch of various sanity checks on the geometry optimiser. Optimisation is } \\
\text { performed, but starting geometries with slightly high energies will not cause } \\
\text { calculation to be rejected. }\end{array}$ \\
\hline MMOK & $\begin{array}{l}\text { Apply a molecular mechanics potential to increase the barrier to rotation of per } \\
\text { bonds (to enforce correct geometries) }\end{array}$ \\
\hline NOINTER & Don't print the interatomic distance numbers in the output files. \\
\hline PM3 & Use the PM3 hamiltonian \\
\hline $\mathrm{T}=120$ & $\begin{array}{l}\text { Halt the optimisation after two minutes if a minimum energy structure has not } \\
\text { been achieved. }\end{array}$ \\
\hline CHARGE $=x$ & Inform MOPAC of the overall charge present on the molecule. \\
\hline
\end{tabular}

\section{Molecular Force Field}

The following parameters define the molecular force field used in the optimisation part of the code. These parameters have been hand-adjusted to obtain reasonable results for many unusual cases. It must be borne in mind that the field is not adjusted to obtain correct molecule geometries directly, but to robustly obtain structures sufficiently reasonable for a quantum mechanics package to work with. Many parameters were derived from data on the NCSA Chemviz website ${ }^{21}$. 
Table 4. Atomic radii, valencies and minimum/maximum charge boundaries for force field employed.

\begin{tabular}{|l|l|l|l|l|}
\hline Element & Radius / $\AA$ & Valency & $\begin{array}{l}\text { Minimum } \\
\text { Charge }\end{array}$ & $\begin{array}{l}\text { Maximum } \\
\text { Charge }\end{array}$ \\
\hline $\mathrm{C}$ & 1.35 & 4 & 0 & 0 \\
\hline $\mathrm{H}$ & 0.85 & 1 & 0 & 0 \\
\hline $\mathrm{N}$ & 1.15 & 3 & 0 & 1 \\
\hline $\mathrm{O}$ & 1.05 & 2 & -1 & 0 \\
\hline $\mathrm{Cl}$ & 1.45 & 1 & 0 & 0 \\
\hline $\mathrm{F}$ & 1 & 1 & 0 & 0 \\
\hline $\mathrm{Br}$ & 1.7 & 1 & 0 & 0 \\
\hline $\mathrm{P}$ & 1.55 & 3 & -2 & 1 \\
\hline $\mathrm{S}$ & 1.5 & 6 & -2 & 0 \\
\hline
\end{tabular}

Table 5. Atomic bond lengths for force field employed.

\begin{tabular}{|l|l|l|l|}
\hline Element 1 & Element 2 & Bond Order & Distance / \\
\hline $\mathrm{H}$ & $\mathrm{H}$ & 1 & 0.74 \\
\hline $\mathrm{H}$ & $\mathrm{B}$ & 1 & 1.19 \\
\hline $\mathrm{H}$ & $\mathrm{C}$ & 1 & 1.095 \\
\hline $\mathrm{H}$ & $\mathrm{N}$ & 1 & 1.01 \\
\hline $\mathrm{H}$ & $\mathrm{P}$ & 1 & 1.44 \\
\hline $\mathrm{H}$ & $\mathrm{O}$ & 1 & 0.96 \\
\hline $\mathrm{H}$ & $\mathrm{S}$ & 1 & 1.34 \\
\hline
\end{tabular}




\begin{tabular}{|c|c|c|c|}
\hline Element 1 & Element 2 & Bond Order & Distance / $\AA$ \\
\hline $\mathrm{H}$ & $\mathrm{F}$ & 1 & 0.92 \\
\hline $\mathrm{H}$ & $\mathrm{Cl}$ & 1 & 1.27 \\
\hline $\mathrm{H}$ & $\mathrm{Br}$ & 1 & 1.41 \\
\hline $\mathrm{H}$ & I & 1 & 1.61 \\
\hline $\mathrm{C}$ & C & 1 & 1.54 \\
\hline $\mathrm{C}$ & $\mathrm{C}$ & 2 & 1.34 \\
\hline $\mathrm{C}$ & $\mathrm{C}$ & 3 & 1.20 \\
\hline $\mathrm{C}$ & C & $\mathrm{Ar}$ & 1.30 \\
\hline C & $\mathrm{N}$ & 1 & 1.47 \\
\hline $\mathrm{C}$ & $\mathrm{N}$ & 2 & 1.29 \\
\hline $\mathrm{C}$ & $\mathrm{N}$ & 3 & 1.16 \\
\hline $\mathrm{C}$ & $\mathrm{P}$ & 1 & 1.84 \\
\hline C & $\mathrm{O}$ & 1 & 1.43 \\
\hline $\mathrm{C}$ & $\mathrm{O}$ & 2 & 1.20 \\
\hline $\mathrm{C}$ & S & 1 & 1.82 \\
\hline $\mathrm{C}$ & $\mathrm{S}$ & 2 & 1.60 \\
\hline C & $\mathrm{F}$ & 1 & 1.35 \\
\hline C & $\mathrm{Cl}$ & 1 & 1.77 \\
\hline C & $\mathrm{Br}$ & 1 & 1.94 \\
\hline $\mathrm{C}$ & I & 1 & 2.14 \\
\hline $\mathrm{N}$ & $\mathrm{N}$ & 1 & 1.45 \\
\hline $\mathrm{N}$ & $\mathrm{N}$ & 2 & 1.25 \\
\hline
\end{tabular}




\begin{tabular}{|c|c|c|c|}
\hline Element 1 & Element 2 & Bond Order & Distance / $\AA$ \\
\hline $\mathrm{N}$ & $\mathrm{N}$ & 3 & 1.10 \\
\hline $\mathrm{N}$ & $\mathrm{O}$ & 1 & 1.40 \\
\hline $\mathrm{N}$ & $\mathrm{O}$ & 2 & 1.21 \\
\hline $\mathrm{N}$ & $\mathrm{F}$ & 1 & 1.36 \\
\hline $\mathrm{N}$ & $\mathrm{Cl}$ & 1 & 1.75 \\
\hline $\mathrm{P}$ & $\mathrm{P}$ & 1 & 2.21 \\
\hline $\mathrm{P}$ & $\mathrm{O}$ & 1 & 1.63 \\
\hline $\mathrm{P}$ & $\mathrm{O}$ & 2 & 1.50 \\
\hline $\mathrm{P}$ & $\mathrm{S}$ & 2 & 1.86 \\
\hline $\mathrm{P}$ & $\mathrm{F}$ & 1 & 1.54 \\
\hline $\mathrm{P}$ & $\mathrm{Cl}$ & 1 & 2.03 \\
\hline $\mathrm{O}$ & $\mathrm{O}$ & 1 & 1.48 \\
\hline $\mathrm{O}$ & $\mathrm{O}$ & 2 & 1.21 \\
\hline $\mathrm{O}$ & $\mathrm{F}$ & 1 & 1.42 \\
\hline $\mathrm{S}$ & $\mathrm{O}$ & 2 & 1.43 \\
\hline S & $S$ & 1 & 2.05 \\
\hline$S$ & S & 2 & 1.49 \\
\hline$S$ & $\mathrm{~F}$ & 1 & 1.56 \\
\hline S & $\mathrm{Cl}$ & 1 & 2.07 \\
\hline $\mathrm{F}$ & $\mathrm{F}$ & 1 & 1.42 \\
\hline $\mathrm{Cl}$ & $\mathrm{Cl}$ & 1 & 1.99 \\
\hline $\mathrm{Br}$ & $\mathrm{Br}$ & 1 & 2.28 \\
\hline
\end{tabular}


Screenshots of Web Site

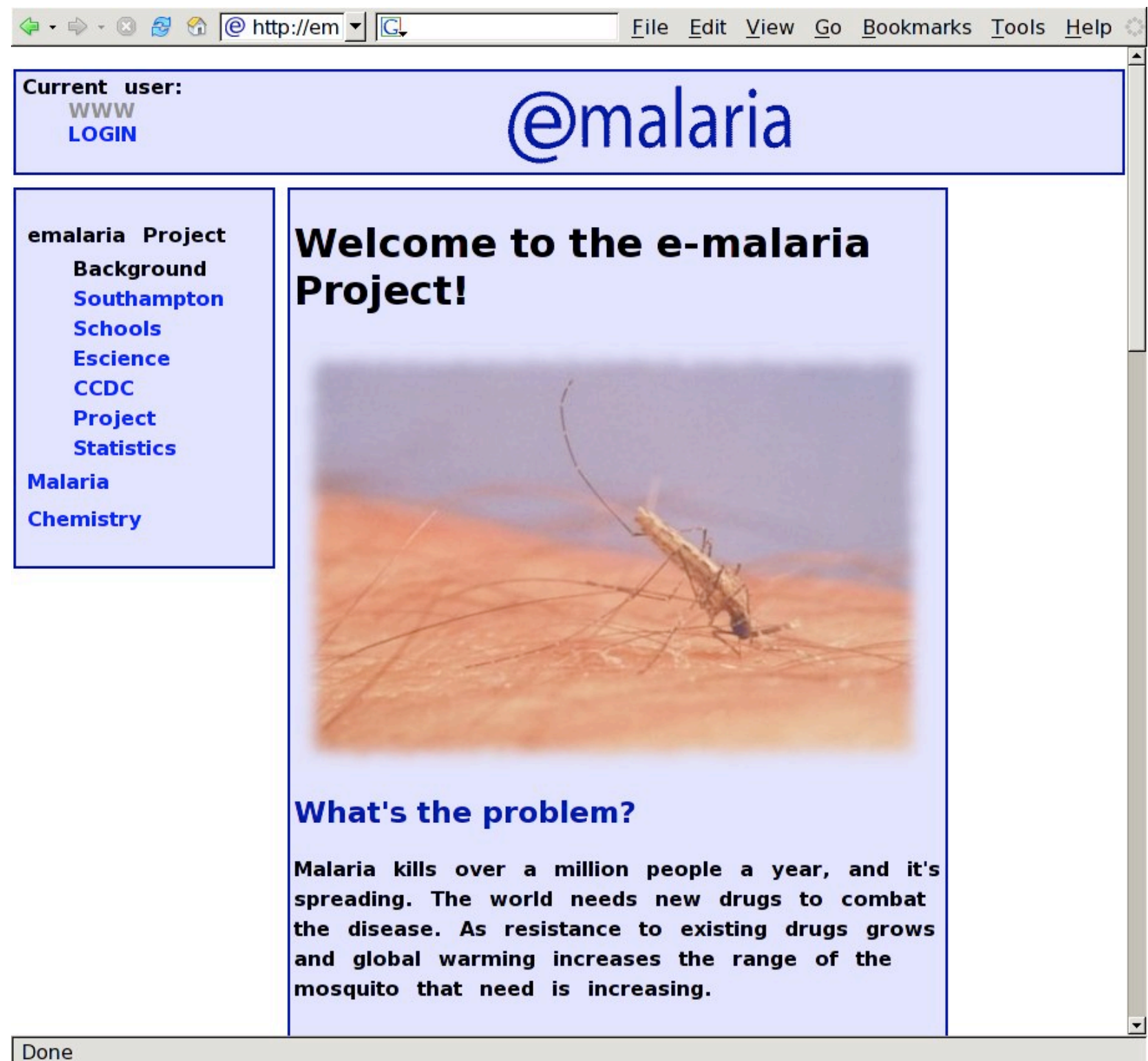




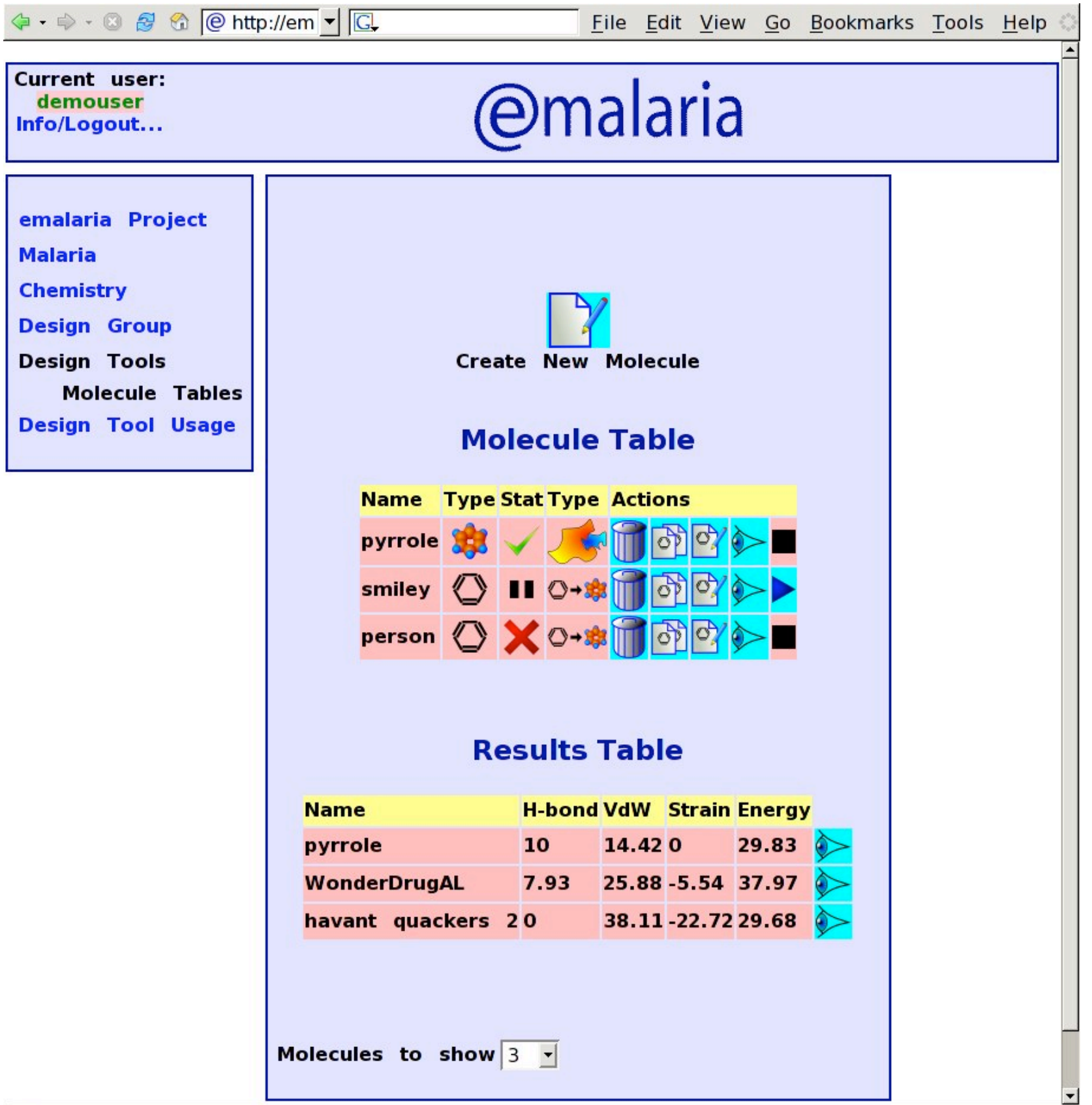




\section{Current user:}

demouser

Info/Logout...

\section{emalaria}

emalaria Project

Malaria

Chemistry

Design Group

Design Tools

Design Tool Usage

\section{Molecule Editor}

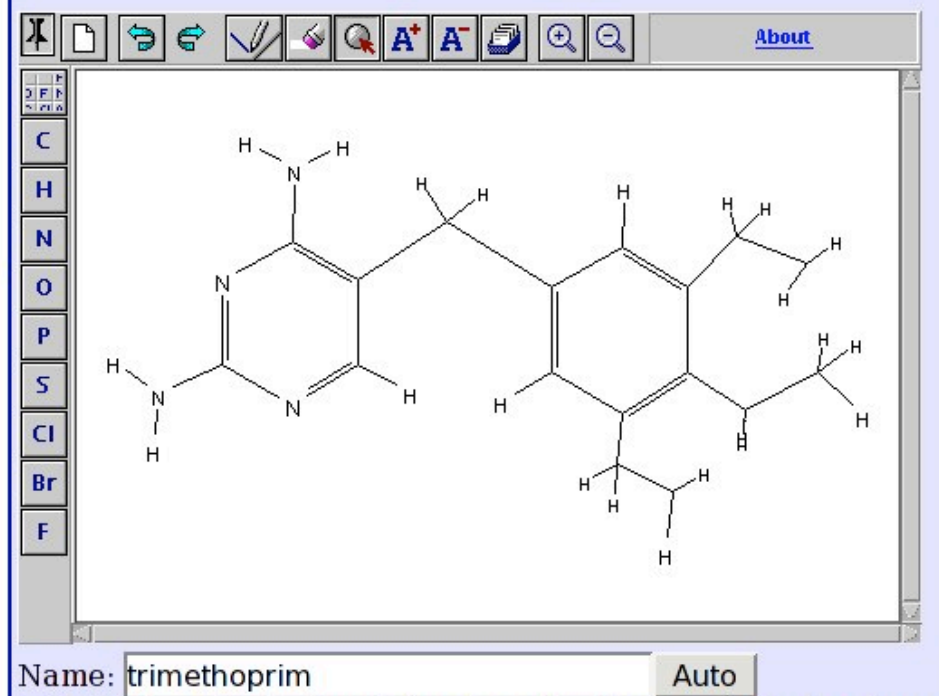

$\ulcorner$ Activate optimisation job automatically on submission (saves you from having to click the "Activate job" triangle in the molecule table)

submit

(C) University of Southampton 2005

For more information, please email emalaria@soton.ac.uk

Applet SDA started 


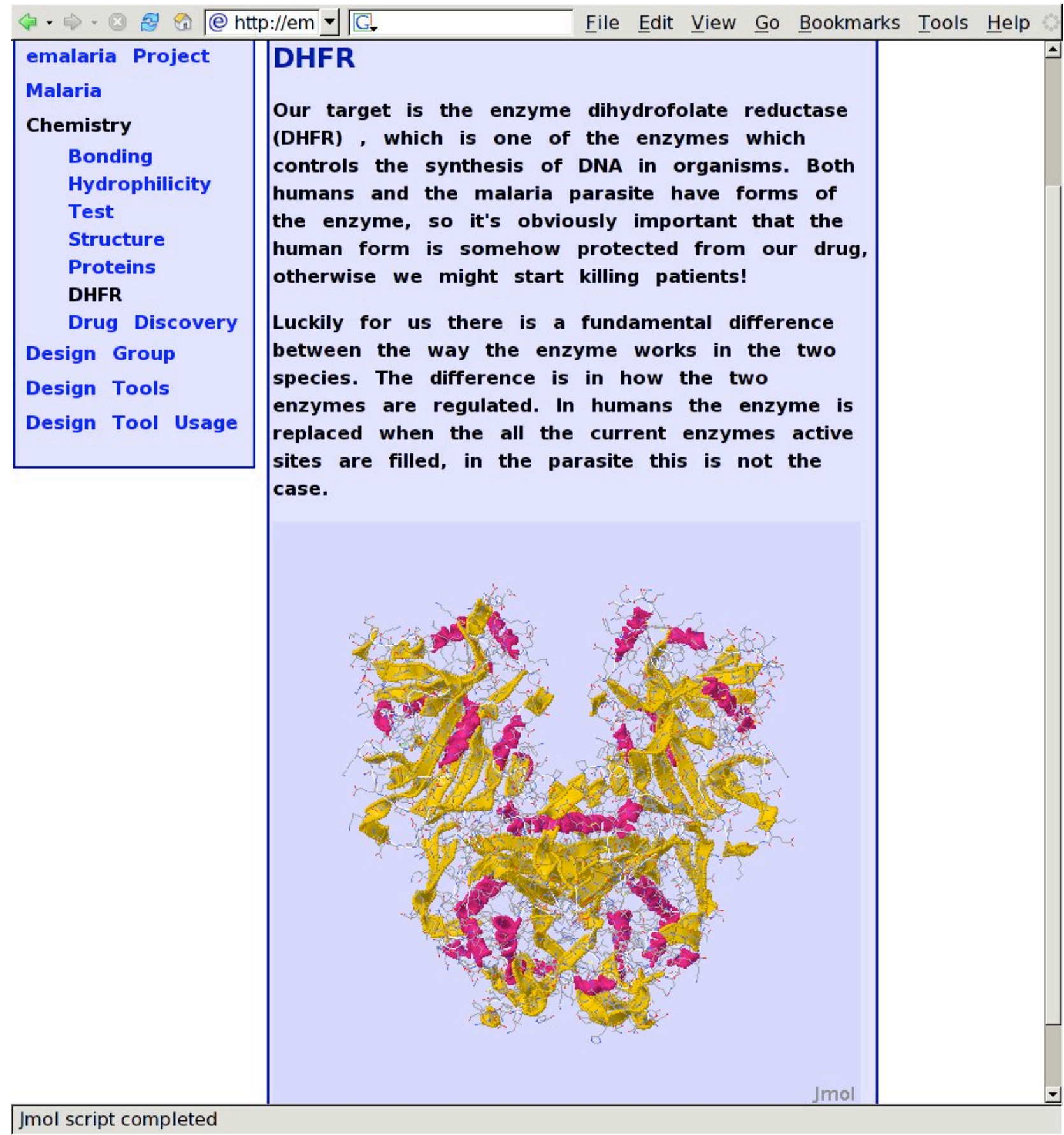

